\title{
Factores que desencadenan las enfermedades hipertensivas en el embarazo en mujeres menores de 20 años atendidas en un hospital de segundo nivel de Hidalgo
}

\author{
Factors that Cause Hypertensive Diseases in Pregnancy in Women Under 20 Years Old \\ Attended at Second Level Hospital of Hidalgo
}

Arias-Rico $J^{a}$, Jiménez-Sánchez $R C^{b}$, Calva-León $E^{c}$, Ramírez-Moreno ${ }^{d}{ }^{d}$, Barrera-Gálvez $R$ ${ }^{e}$, Ramírez-Jiménez EV ${ }^{f}$, Alcántara-de-Rosas $L^{f}$, Cruz-Hernández DI ${ }^{f}$, García-Fragoso $O^{f}$ , Guzmán- de la Rosa $P^{f}{ }^{\text {, Luna- Jiménez ER }}{ }^{f}$

\begin{abstract}
:
The objective of this work was to identify the factors that trigger hypertensive diseases in pregnancy at an early age. Methodology. This study is of a correlational type since the predisposing factors for the presence of hypertensive diseases in pregnant women under 20 years old are related, which seeks to relate each factor with the classification of hypertensive diseases. Results. From a universe of 50 pregnant women, a total of $72 \%$ women were obtained whose occupation is housewife, $24 \%$ student, $4 \%$ employed. $0 \%$ without studies, 68\% basic level, 32\% upper middle level. 44\% know that it is hypertension, and the remaining 56\% do not know. Discussion. According to Viviana Sáez in her study of pre-eclampsia, arterial hypertension had an incidence of great importance since $68 \%$ of pregnant adolescents reported having more than 3 signs and symptoms of hypertension during the development of their pregnancy. Conclusion. The factors that trigger hypertensive diseases in pregnancy are primarily the hereditary family history, and nutritional disorders prior to pregnancy, such as overweight.
\end{abstract}

\section{Keywords:}

Placental abruption, Eclampsia, Edema, Hemorrhage Preeclampsia, Proteinuria, Puerperium, Phosphenes, Cytokines, Chorioamionitis

\section{Resumen:}

El objetivo de este trabajo fue identificar los factores que desencadenan las enfermedades hipertensivas en el embarazo a temprana edad. Metodología. Este estudio es de tipo correlacional ya que se relacionaran los factores predisponentes para la presencia de enfermedades hipertensivas en embarazadas menores de 20 años que busca relacionar cada factor con la clasificación de enfermedades hipertensivas. Resultados. De universo de 50 mujeres embarazadas, se obtuvo un total de $72 \%$ mujeres cuya ocupación es ama de casa, 24\% estudiante, 4\% empleada. El $0 \%$ sin estudios, $68 \%$ nivel básico, 32\% nivel medio superior. El 44\% conoce que es hipertensión arterial, y el 56\% restante desconoce. Discusión. Según Viviana Sáez en su estudio la pre-eclampsia, la hipertensión arterial tuvo una incidencia de gran importancia puesto que el 68\% de adolescentes embarazadas refirieron tener más de 3 signos y síntomas de HTA durante el desarrollo de su embarazo. Conclusión. Los factores que desencadenan las enfermedades hipertensivas en el embarazo son en primera instancia los antecedentes patológicos heredofamiliares, y los trastornos nutricionales previos al embarazo, tal como sobrepeso.

\section{Palabras Clave:}

Abrupción placentaria, Eclampsia, Edema, Hemorragia Preeclampsia, Proteinuria, Puerperio, Fosfenos, Citoquinas, Corioamionitis

\footnotetext{
a Autor de Correspondencia, Universidad Autónoma del Estado de Hidalgo, Instituto de Ciencias de la Salud, ORCID: https://orcid.org/00000003-0219-0410, Email: josearias.rico@hotmail.com

${ }^{\mathrm{b}}$ Universidad Autónoma del Estado de Hidalgo, Instituto de Ciencias de la Salud, ORCID: https://orcid.org/0000-0001-9264-8514, Email: cristiji@hotmail.com

c Universidad Autónoma del Estado de Hidalgo, Instituto de Ciencias de la Salud, Email: lic.eduardo.uaeh@gmail.com

d Universidad Autónoma del Estado de Hidalgo, Instituto de Ciencias de la Salud, ORCID: https://orcid.org/0000-0002-9928-8600, Email: rme1234@yahoo.com

e Universidad Autónoma del Estado de Hidalgo, Instituto de Ciencias de la Salud, ORCID: https://orcid.org/0000-0002-1949-5424, Email: rosario_barrera@uaeh.edu.mx

${ }^{\mathrm{f}}$ Alumnos de la Universidad Autónoma del Estado de Hidalgo, Instituto de Ciencias de la Salud, Email: josearias.rico@hotmail.com
} 


\section{Introducción}

Este tema es de gran importancia en la investigación ya que dada su incidencia en la morbi-mortalidad materna, cobra una importancia decisiva especialmente en mujeres jóvenes. Se revisa lo relacionado con la Preeclampsia, Eclampsia, y síndrome de HELLP, haciendo hincapié en aquellos aspectos relacionados con la atención a la paciente grave en este tipo de características. El surgimiento de prevención en la atención al paciente grave ha podido disminuir la mortalidad y las complicaciones mediante la vigilancia y el empleo de modernas técnicas diagnósticas y terapéuticas.

Este enfoque es muy importante ya que corre el riesgo de sufrir daños en su mayoría de las ocasiones irreparables que podrían resultar mortales afectando la vida de este binomio madre y producto gestante, además que es de gran importancia el preservar la integridad de este tipo de pacientes.

El embarazo es un evento fisiológico en la mujer, el cual es una causa de autorrealización en ella y de felicidad en la familia, aunque en algunas ocasiones este se convierte en una causa de preocupación, estrés, miedo, ya que en la actualidad la tasa de muerte materna en el mundo es relativamente alta, según la OMS, 2015, cada día mueren cerca de 830 mujeres en el mundo por causas relacionadas al embarazo, parto y/o puerperio, muchas de estas son prevenibles; entre las causas más comunes y de mayor prevalencia se encuentra, las hemorragias obstétricas, infecciones postparto, complicaciones relacionadas con el parto, abortos, y otra más y de mucha importancia son las enfermedad hipertensiva en el embarazo [1].

De acuerdo con la OMS, el porcentaje de mortalidad materna por causa es de: enfermedades persistentes y agravadas por el embarazo es de $28 \%$, hemorragias $27 \%$, hipertensión inducida por el embarazo 14\%, infecciones postparto $11 \%$, complicaciones durante el parto $9 \%$, complicaciones de un aborto $8 \%$, embolias $3 \%$; de acuerdo a esto la HAS gestacional es una de las tres principales causas de mortalidad en mujeres en edad reproductiva; en el 2010 [2]. México es el estado que obtuvo el porcentaje de muertes a causa hipertensivas con un $11.7 \%$, después le sigue Chiapas, distrito federal y Veracruz con un $7.7 \%$, los estados con los porcentajes más bajos de muertes por estas causas son Morelos y Querétaro con un $0.4 \%$, los estados que no registran muertes (muertes por la misma causa) son baja california sur, Campeche, colima, Nayarit y sonora [3,4].

El objetivo de este trabajo fue identificar cuáles son las principales causas por las que se presentan enfermedades hipertensivas en el embarazo a una edad temprana, ya que la hipertensión en madres jóvenes es la complicación médica más frecuente en el embarazo, siendo una causa significativa de prematuros y morbimortalidad perinatal, es además una de las primeras causas de muerte materna. Esta problemática no solo está presente en los hospitales, sino que también en el primer nivel de atención, donde en algunas ocasiones son detectadas. De igual manera consideramos que los embarazos a temprana edad es una problemática de planificación familiar y de educación e información deficiente, que, si nosotros como profesionales de salud trabajamos en conjunto con padres de familia, maestros algunos otros profesionales, podemos reducir el índice de embarazos en la adolescencia y por consiguiente prevenir y evitar las complicaciones en madres jóvenes.

\section{Metodología}

El tipo de investigación para este estudio es de tipo correlacional, de diseño transversal, con criterios de inclusión: Asistir o recibir atención en el hospital de segundo nivel del estado de Hidalgo, que sea menor de 20 años de edad, mujer que esté embarazada y curse con preclamsia, que desee participar voluntariamente en el estudio. Se utilizó un instrumento de medición de 25 ítems y con un alfa de Crombach de 0.738. El estudio estuvo apegado a los parámetros de Ética del comité del Instituto de Ciencias de la Salud, UAEH.

\section{Resultados}

Se registraron 135 casos mujeres con Morbilidad Materna Extrema, la edad promedio encontrada fue de 26 años; el $54.8 \%$ de las mujeres presentó múltiples embarazos (mujeres multigestas). La mayoría de los casos, el 83.7\%, presentó morbilidades por causas obstétricas directas tales como Preeclampsia, Hemorragia y Sepsis; mientras que el $16.2 \%$ presentó morbilidades por causas indirectas. De los 135 casos, se encontró que 120 de las mujeres derivaron en secuelas agudas (81.6\%) y en crónicas (18.3\%).

Factores condicionantes básicos hace referencia a los factores que condicionan o alteran el valor de la demanda de autocuidado terapéutico y/o la actividad de autocuidado de un individuo en momentos concretos y bajo circunstancias específicas. Se han identificado diez factores:

El tipo de investigación para este estudio es de tipo correlacional ya que se relacionarán los factores predisponentes para la presencia de enfermedades hipertensivas en embarazadas menores de 20 años, esto sería la variable independiente y se buscará relacionar cada factor (variable independiente) con la clasificación de enfermedades hipertensivas, Hipertensión arterial, Preclamsia, eclampsia y síndrome de Hellp que vendrían siendo las variables dependientes.

Para la realización de los datos obtenidos en la aplicación del presente instrumento de valoración se realizó la aplicación de las encuestas en el Hospital de segundo nivel de atención, específicamente en el servicio de módulo mater, urgencias ginecológicas, y la consulta externa del mismo hospital anteriormente mencionado, en una jornada matutina completa de 8 horas. De este modo se obtuvo un universo de 50 mujeres embarazadas, de las cuales $50 \%(n=25)$ de las mujeres encuestadas no entraban dentro de los parámetros establecidos para la realización de esta investigación, por lo cual fueron excluidas de la muestra significativa total para el análisis de la muestra mencionada. La aplicación de los 
resultados donde se introdujeron las variables que se analizaron a la previa realización del presente trabajo de investigación.

Es por ello que entonces se tomaron solamente las 25 mujeres que cumplían con los criterios de inclusión establecidos de este modo se decidió tomar una muestra por conveniencia de estas 25 mujeres embarazadas menores de 20 años (tabla 1).

Tabla 1. Edad de la muestra.

\begin{tabular}{|l|l|l|}
\hline & Mujeres & Total \%(n) \\
\hline Total & $100 \%(n=25)$ & $100 \%(n=25)$ \\
\hline \multicolumn{3}{|c|}{1.1 EDAD } \\
\hline 14 AÑOS & $4 \%(n=1)$ & $4 \%(n=1)$ \\
\hline 15 AÑOS & $8 \%(n=2)$ & $8 \%(n=2)$ \\
\hline 16 AÑOS & $12 \%(n=3)$ & $12 \%(n=3)$ \\
\hline 17 AÑOS & $12 \%(n=3)$ & $12 \%(n=3)$ \\
\hline 18 AÑOS & $16 \%(n=4)$ & $16 \%(n=4)$ \\
\hline 19 AÑOS & $16 \%(n=4)$ & $16 \%(n=4)$ \\
\hline 20 AÑOS & $32 \%(n=8)$ & $32 \%(n=8)$ \\
\hline
\end{tabular}

El $72 \%$ mujeres cuya ocupación es ama de casa, $24 \%$ estudiante, $4 \%$ empleada de la misma manera se evaluó el nivel de estudios de: $0 \%$ sin estudios, $68 \%$ nivel básico, $32 \%$ nivel medio superior. (Tabla 2 ).

Tabla 2. Ocupación de los sujetos de estudio

\begin{tabular}{|c|c|c|}
\hline & Mujeres & Total \%(n) \\
\hline Estudiante & $24 \%(n=6)$ & $24 \%(n=6)$ \\
\hline $\begin{array}{c}\text { Ama de } \\
\text { casa }\end{array}$ & $\begin{array}{c}72 \%(n=18 \\
)\end{array}$ & $72 \%(\mathrm{n}=18)$ \\
\hline Empleada & $4 \%(\mathrm{n}=1)$ & $4 \%(\mathrm{n}=1)$ \\
\hline \multicolumn{2}{|c|}{ Nivel de estudios } \\
\hline $\begin{array}{c}\text { Sin } \\
\text { estudios }\end{array}$ & $0 \%(\mathrm{n}=0)$ & $0 \%(\mathrm{n}=0)$ \\
\hline $\begin{array}{c}\text { Nivel } \\
\text { básico }\end{array}$ & $68 \%(\mathrm{n}=17$ & $68 \%(\mathrm{n}=17)$ \\
\hline $\begin{array}{c}\text { Nivel } \\
\text { medio } \\
\text { superior }\end{array}$ & $32 \%(\mathrm{n}=8)$ & $32 \%(\mathrm{n}=8)$ \\
\hline $\begin{array}{c}\text { Nivel } \\
\text { superior }\end{array}$ & $0 \%(\mathrm{n}=0)$ & $0 \%(\mathrm{n}=0)$ \\
\hline
\end{tabular}

Tabla 3. Conoce la hipertensión arterial en el embarazo

\begin{tabular}{|l|l|l|}
\hline & Mujeres & Total \%(n) \\
\hline si & $44 \%(n=11)$ & $44 \%(n=11)$ \\
\hline no & $56 \%(n=14)$ & $56 \%(n=14)$ \\
\hline \multicolumn{3}{|c|}{ Conoce la Eclampsia y Preclampsia } \\
\hline Si & $60 \%(n=15)$ & $60 \%(n=15)$ \\
\hline No & $40 \%(n=10)$ & $40 \%(n=10)$ \\
\hline
\end{tabular}

De la misma manera en cuestión de conocimiento y familiarización con la y terminología se obtiene $44 \%$ conoce qué es hipertensión arterial, y el 56\% restante desconoce el significado, así como los síntomas y signos que se manifiestan en cada una de estas patologías. (Tabla 3).

Tabla 4. En qué trimestre del embarazo se encuentra.

\begin{tabular}{|c|c|c|}
\hline & Mujeres & Total \%(n) \\
\hline Primer trimestre & $24 \%(n=6)$ & $24 \%(n=6)$ \\
\hline $\begin{array}{l}\text { Segundo } \\
\text { trimestre }\end{array}$ & $28 \%(n=7)$ & $28 \%(n=7)$ \\
\hline Tercer trimestre & $48 \%(n=12)$ & $48 \%(n=12)$ \\
\hline \multicolumn{3}{|c|}{$\begin{array}{l}\text { 1.9 Ha tenido atención del primer contacto en algún } \\
\text { servicio de salud? }\end{array}$} \\
\hline $\mathrm{Si}$ & $84 \%(n=21)$ & $84 \%(n=21)$ \\
\hline No & $16 \%(n=4)$ & $16 \%(n=4)$ \\
\hline \multicolumn{3}{|c|}{ Lleva control prenatal periódicamente } \\
\hline $\mathrm{Si}$ & $96 \%(n=24)$ & $96 \%(n=24)$ \\
\hline No & $4 \%(n=1)$ & $4 \%(n=1)$ \\
\hline \multicolumn{3}{|c|}{ Cuántas consultas lleva hasta este momento } \\
\hline $1-3$ & $20 \%(n=5)$ & $20 \%(n=5)$ \\
\hline $4-6$ & $32 \%(\mathrm{n}=8)$ & $32 \%(\mathrm{n}=8)$ \\
\hline Más de 6 & $48 \%(n=12)$ & $48 \%(\mathrm{n}=12)$ \\
\hline Ninguna & $0 \%(\mathrm{n}=0)$ & $0 \%(\mathrm{n}=0)$ \\
\hline
\end{tabular}

Por otro lados se tiene que el $100 \%$ de las mujeres embarazadas conoce en que trimestre del embarazo se encuentra, más sin embargo $84 \%$ ha tenido atención de primer contacto en algún servicio de salud, así mismo 96\% lleva control prenatal periódicamente, es por ello que no podemos percatar de que un \% del total de las mujeres entrevistadas tiene más de 6 consultas, que es la cantidad mínima exigida por la Cartilla Nacional de salud y la (Norma Oficial Mexicana) NOM 007-SSA2-2016 [6], Para la atención de la mujer durante el embarazo, y puerperio, y de la persona recién nacida. (Tabla 4). 
Tabla 5. Tiene el apoyo de la familia

\begin{tabular}{|c|c|c|}
\hline & Mujeres & Total \%(n) \\
\hline $\mathrm{Si}$ & $96 \%(\mathrm{n}=24)$ & $96 \%(\mathrm{n}=24)$ \\
\hline $\mathrm{No}$ & $4 \%(\mathrm{n}=1)$ & $4 \%(\mathrm{n}=1)$ \\
\hline \multicolumn{3}{|c|}{ Tienes el apoyo de tu pareja } \\
\hline $\mathrm{Si}$ & $80 \%(\mathrm{n}=20)$ & $80 \%(\mathrm{n}=20)$ \\
\hline $\mathrm{No}$ & $20 \%(\mathrm{n}=5)$ & $20 \%(\mathrm{n}=5)$ \\
\hline
\end{tabular}

En cuanto al apoyo familiar y de pareja nos percatamos de que si cuentan con este apoyo en un $96 \%$ del total de las entrevistadas. (Tabla 5). En cuestión de salud la relación en ganancia de peso en relación con el trimestre en el cual se encuentran es superior a los 12 kilos durante el periodo gestacional es de $8 \%$, y en otro apartado referido en salud personal se obtiene que un $96 \%$ conoce y menciona signos y síntomas de alarma, por otro lado las mujeres embarazadas tienen familiares con hipertensión arterial (familiares hombres y/o mujeres) del $100 \%$ de las participantes en la investigación 20\% tienen familiares directos (mujeres) que padecieron enfermedades hipertensivas en el embarazo. (Tabla 6).

Tabla 6. Cuántos kilos ha aumentado de peso durante tu embarazo.

\begin{tabular}{|c|c|c|}
\hline 1-4 kilos & $28 \%(n=7)$ & $28 \%(\mathrm{n}=7)$ \\
\hline 5-8 kilos & $12 \%(n=3)$ & $12 \%(n=3)$ \\
\hline 9-12 kilos & $52 \%(\mathrm{n}=13)$ & $52 \%(n=13)$ \\
\hline $\begin{array}{l}\text { Más de } 12 \\
\text { kilos }\end{array}$ & $8 \%(\mathrm{n}=2)$ & $8 \%(n=2)$ \\
\hline \multicolumn{3}{|c|}{ Conoce los síntomas y signos de alarma en el embarazo } \\
\hline $\mathrm{Si}$ & $96 \%(n=24)$ & $96 \%(n=24)$ \\
\hline No & $4 \%(n=1)$ & $4 \%(n=1)$ \\
\hline \multicolumn{3}{|c|}{$\begin{array}{l}\text { Cuántas veces ha presentado signos y síntomas de alarma } \\
\text { en tu embarazo }\end{array}$} \\
\hline 1 & $28 \%(n=7)$ & $28 \%(n=7)$ \\
\hline 2 & $4 \%(n=1)$ & $4 \%(n=1)$ \\
\hline 3 & $4 \%(n=1)$ & $4 \%(n=1)$ \\
\hline 4 & $12 \%(n=3)$ & $12 \%(n=3)$ \\
\hline Más de 5 & $52 \%(n=13)$ & $52 \%(n=13)$ \\
\hline \multicolumn{3}{|c|}{ Tiene familiares directos que tengan hipertensión arterial } \\
\hline $\mathrm{Si}$ & $20 \%(n=5)$ & $20 \%(n=5)$ \\
\hline No & $80 \%(n=20)$ & $80 \%(n=20)$ \\
\hline \multicolumn{3}{|c|}{$\begin{array}{l}\text { Tiene familiares mujeres que hayan tenido hipertensión } \\
\text { arterial durante su embarazo }\end{array}$} \\
\hline $\mathrm{Si}$ & $20 \%(n=5)$ & $20 \%(n=5)$ \\
\hline No & $80 \%(n=20)$ & $80 \%(n=20)$ \\
\hline
\end{tabular}

Tabla 7. Cuántos días a la semana consume harinas y alimentos enlatados.

\begin{tabular}{|c|c|c|}
\hline Ningún día & $20 \%(\mathrm{n}=5)$ & $20 \%(\mathrm{n}=5)$ \\
\hline $2-3$ días & $76 \%(\mathrm{n}=19)$ & $76 \%(\mathrm{n}=19)$ \\
\hline $4-5$ días & $4 \%(\mathrm{n}=1)$ & $4 \%(\mathrm{n}=1)$ \\
\hline Diario & $0 \%(\mathrm{n}=0)$ & $0 \%(\mathrm{n}=0)$ \\
\hline \multicolumn{3}{|c|}{ Consume ácido fólico } \\
\hline $\mathrm{Si}$ & $92 \%(\mathrm{n}=23)$ & $92 \%(\mathrm{n}=23)$ \\
\hline $\mathrm{No}$ & $8 \%(\mathrm{n}=2)$ & $8 \%(\mathrm{n}=2)$ \\
\hline \multicolumn{3}{|c|}{ Consume hierro } \\
\hline $\mathrm{Si}$ & $92 \%(\mathrm{n}=23)$ & $92 \%(\mathrm{n}=23)$ \\
\hline $\mathrm{No}$ & $8 \%(\mathrm{n}=2)$ & $8 \%(\mathrm{n}=2)$ \\
\hline \multicolumn{3}{|c|}{ Consumo de multivitamínico } \\
\hline $\mathrm{Si}$ & $40 \%(\mathrm{n}=10)$ & $40 \%(\mathrm{n}=10)$ \\
\hline $\mathrm{No}$ & $60 \%(\mathrm{n}=15)$ & $60 \%(\mathrm{n}=15)$ \\
\hline
\end{tabular}

También se pudo valorar el estado nutricional de las pacientes encontrando que un $76 \%$ consume alimentos enlatados y harinas con regularidad, un $92 \%$ consume ácido fólico y hierro durante la gestación un 40\% multivitamínico (Tabla 7).

Tabla 8. Cuántos días a la semana consume carne.

\begin{tabular}{|c|c|c|}
\hline Ningún día & $8 \%(n=2)$ & $8 \%(n=2)$ \\
\hline 2-3dias & $60 \%(n=15)$ & $60 \%(n=15)$ \\
\hline 4-6 días & $32 \%(n=8)$ & $32 \%(\mathrm{n}=8)$ \\
\hline Diario & $0 \%(\mathrm{n}=0)$ & $0 \%(\mathrm{n}=0)$ \\
\hline \multicolumn{3}{|c|}{ Cuántos días a la semana consume frutas. } \\
\hline Ningún día & $0 \%(n=0)$ & $0 \%(\mathrm{n}=0)$ \\
\hline 2-3dias & $20 \%(n=5)$ & $20 \%(n=5)$ \\
\hline 4-6 días & $12 \%(n=3)$ & $12 \%(n=3)$ \\
\hline Diario & $68 \%(n=17)$ & $68 \%(n=17)$ \\
\hline \multicolumn{3}{|c|}{ Cuántos días a la semana consume verduras. } \\
\hline Ningún día & $0 \%(\mathrm{n}=0)$ & $0 \%(\mathrm{n}=0)$ \\
\hline 2-3dias & $28 \%(n=7)$ & $28 \%(n=7)$ \\
\hline 4-6 días & $16 \%(n=4)$ & $16 \%(n=4)$ \\
\hline Diario & $56 \%(n=14)$ & $56 \%(n=14)$ \\
\hline \multicolumn{3}{|c|}{ Cuántos días a la semana consume semillas } \\
\hline Ningún día & $12 \%(n=3)$ & $12 \%(n=3)$ \\
\hline 2-3dias & $48 \%(n=12)$ & $48 \%(n=12)$ \\
\hline 4-6 días & $12 \%(n=3)$ & $12 \%(n=3)$ \\
\hline Diario & $28 \%(n=7)$ & $28 \%(n=7)$ \\
\hline \multicolumn{3}{|c|}{ Cantidad de agua ingiere al día. } \\
\hline $\begin{array}{c}\text { Menos de } 1 \\
\text { litro }\end{array}$ & $4 \%(\mathrm{n}=1)$ & $4 \%(n=1)$ \\
\hline 1 litro & $48 \%(n=12)$ & $48 \%(n=12)$ \\
\hline $\begin{array}{c}\text { De } 2 \text { a } 3 \\
\text { litros }\end{array}$ & $48 \%(n=12)$ & $48 \%(n=12)$ \\
\hline \multicolumn{3}{|c|}{ Frecuencia de agregar sal a los alimentos que consume } \\
\hline Siempre & $4 \%(n=1)$ & $4 \%(n=1)$ \\
\hline $\begin{array}{l}\text { Algunas } \\
\text { veces }\end{array}$ & $36 \%(n=9)$ & $36 \%(n=9)$ \\
\hline Rara vez & $36 \%(n=9)$ & $36 \%(n=9)$ \\
\hline $\begin{array}{c}\text { No consumo } \\
\text { sal }\end{array}$ & $24 \%(n=6)$ & $24 \%(n=6)$ \\
\hline
\end{tabular}


Así mismo se evaluó la cantidad de alimentos que comen de manera semanal encontrando lo siguiente: un $60 \%$ consume carne $2 / 3$ días por semana, $68 \%$ consumen frutas diarias y un $56 \%$ consume verduras diariamente, un $48 \%$ consume semillas $2 / 3$ dias a la semana, En cuanto a la ingesta de agua durante el día se obtuvo un $48 \%$ que consume más de 1 lts, $48 \%$ 2-3lts, así mismo un $36 \%$ agrega sal extra a los alimentos que consume con regularidad (Tabla 8 ).

Tabla 9. Antecedente de padecer sobrepeso u obesidad en el embarazo actual.

\begin{tabular}{|l|c|c|}
\hline Si & $28 \%(n=7)$ & $28 \%(\mathrm{n}=7)$ \\
\hline No & $72 \%(\mathrm{n}=18)$ & $72 \%(\mathrm{n}=18)$ \\
\hline \multicolumn{2}{|c|}{ Conoce tus últimas cifras de presión arterial. } \\
\hline SI & $56 \%(\mathrm{n}=14)$ & $56 \%(\mathrm{n}=14)$ \\
\hline No & $44 \%(\mathrm{n}=16)$ & $44 \%(\mathrm{n}=16)$ \\
\hline
\end{tabular}

$\mathrm{Y}$ en cuanto al estado nutricional previo al embarazo se obtiene un $28 \%$ que padeció algún problema de desnutrición u obesidad previa al embarazo. De este modo por razones lógicas desconoce un $44 \%$ cuáles son sus 3 últimas cifras de presión arterial. (Tabla 9).

\section{Discusión}

Según Viviana Sáez en su estudio la pre-eclampsia incidió en adolescentes en un 6,8 \%, en cuanto a la investigación realizada, la hipertensión arterial tuvo una incidencia de gran importancia puesto que el 68\% de adolescentes embarazadas refirieron tener más de 3 signos y síntomas de HTA durante el desarrollo de su embarazo [7]. Sigfrido Valdés y colaboradores encontraron que la pre-eclampsia predominó en menores de 19 años con el $22.5 \%$, teniendo un universo de 40 embarazadas. Se ha demostrado una incidencia importante de enfermedades hipertensivas en el embarazo en adolescentes puesto que en las investigaciones las adolescentes ocupan un número notorio con riesgo de alguna enfermedad hipertensiva [8]. De acuerdo a Viviana Sáez, es muy frecuente que en el embarazo sobre todo en mujeres con dieta inadecuada, como el caso de las menores de 20 años, donde los caprichos y malos hábitos alimentarios propios de la edad contribuyen a un déficit de nutrientes, mismas deficiencias se encontraron en la investigación realizada ya que el $28 \%$ de las encuestadas padecieron sobrepeso u obesidad y un $4 \%$ desnutrición, antes o durante el desarrollo del embarazo, además el $76 \%$ tienen un consumo excesivo de productos enlatados y harinas, además un $60 \%$ de las encuestadas tiene un alto consumo de carne lo que condiciona un factor de riesgo para desencadenar una enfermedad hipertensiva en el embarazo debido a la mala alimentación [7], y teniendo en cuenta que hay una gran inmadurez en el desarrollo fisiológico y anatómico de las adolescentes, según datos del 2013 del INE (Instituto Nacional de Estadística), la edad media de las madres primerizas es de 32,2 años. En el estudio de Yaneth Borregales, estuvo integrada mayormente por adolescentes de 17 a 19 años. Estos datos coinciden con la investigación, sólo que con un ligero incremento de la edad, ya que el mayor grupo de edad en las adolescentes reportada en nuestra investigación fue de 20 años con un 32\%, lo que quiere decir que la edad de embarazos está aumentando de los 20 años y no menor de estos $[9,10,11]$.

\section{Conclusiones}

En ésta investigación se encontró que las mujeres embarazadas desconocen las enfermedades hipertensivas en el embarazo (56\%), por esta misma razón hacen caso omiso a la aparición de signos y síntomas. Se demostró que el $96 \%$ de las mujeres embarazadas encuestadas mencionan conocer los signos y síntomas de alarma además de referir que el $68 \%$ de ellas han presentado más de 3 signos y síntomas aun llevando un control prenatal periódicamente.

En los resultados obtenidos en esta investigación permite realizar una lista de los factores que influyen en el desencadenamiento de las enfermedades hipertensivas en el embarazo en las mujeres menores de 20 años que son atendidas en el Hospital de Segundo Nivel del Estado de Hidalgo; estos factores son: la inmadurez en el desarrollo fisiológico y anatómico de la mujer, los trastornos alimenticios o los malos hábitos de alimentación que se tiene en esta zona, ya que la comida que se consume es pobre en nutrientes, otro de los factores son los trastornos nutricionales antes del embarazo como obesidad y sobrepeso. De igual manera se encontró que los desórdenes nutricionales como la obesidad y el sobrepeso previos al embarazo, si influyen para la aparición de signos y síntomas de enfermedades hipertensivas en el embarazo, de igual manera la calidad de la alimentación durante la gravidez, del total de las mujeres encuestadas el $28 \%$ presentaron obesidad previa al embarazo y de estas mismo el $28 \%$ presento signos y síntomas de alarma.

\section{Referencias}

[1]. Asanza, K. C., Sánchez, C. E. P., \& Meneses, C. A. Morbilidad materna extremadamente grave y mortalidad, indicadores de calidad de la atención obstétrica. MULTIMED, 2017, 21(3), 289-310.

[2]. Changoluisa, T., \& Isabel, A. Conocimientos y prácticas sobre el partopuerperio en las parteras empíricas y su influencia en las complicaciones obstétricas en las comunidades rurales del cantón Salcedo (Bachelor's thesis, Universidad Técnica de Ambato-Facultad de Ciencias de la Salud-Carrera de Medicina). (2015).

[3]. Calvo-Aguilar O, Morales-García VE, Fabián-Fabián J. Morbilidad materna extrema en el Hospital General Dr. Aurelio Valdivieso, Servicios de Salud de Oaxaca. Ginecol Obstet Mex. 2010. 
[4]. Cruz MDCR, Hernández RG, González MG, García GM. Causas, frecuencia y secuelas de la morbilidad materna extrema en mujeres mexicanas: un análisis de los códigos rojos en el IMSS de Hidalgo. Rev CONAMED [Internet]. 2016 Dec 16 [cited 2017 Sep 14];21(4).

[6]. NOM, Norma Oficial Mexicana. 007-SSA2-2016, Para la atención de la mujer durante el embarazo, parto y puerperio, y de la persona recién nacida. Diario Oficial, 2016, vol. 7.

[7].Diago Caballero, Dalis, et al. "Factores de riesgo en la hipertensión inducida por el embarazo." Revista Cubana de Obstetricia y Ginecología 37.4 (2011): 448-456.

[8].Chacón Olivas, Aquiles Gerardo. "Complicaciones maternas perinatales en gestantes adolescentes atendidas en el Hospital Regional de Loreto 2016-2017." (2019).

[9]. Zo, Hipertensión jnducida por el embapj. "universidad del zuua facultad de medicina. 2018.

[10].Huanco AD, Ticona RM, Ticona VM, Huanco AF. Frecuencia y repercusiones maternas y perinatales del embarazo en adolescentes atendidas en hospitales del Ministerio de Salud del Perú, año 2008. Rev Chil Obstet Ginecol. 2012;77(2):122-8.

[11]. Huanco A D, Ticona R M, Ticona V M, Huanco A F. Frecuencia y repercusiones maternas y perinatales del embarazo en adolescentes atendidas en hospitales del Ministerio de Salud del Perú, año 2008. Rev Chil Obstet Ginecol [Internet]. Sociedad Chilena de Obstetricia y Ginecología; 2012 [cited 2017 Sep 14];77(2):122-8 